\title{
Brief Assessment of Cognition in Schizophrenia Functional Test
}

National Cancer Institute

\section{Source}

National Cancer Institute. Brief Assessment of Cognition in Schizophrenia Functional

Test. NCI Thesaurus. Code C121004.

A standardized rating scale developed by Keefe et al in 2004 to assess cog nitive function and impairment in patients with schizophrenia. This instrument consists 6 tests in the following areas: verbal memory, working memory, motor speed, verbal fluency, attention, and executive function. 